\title{
VORWORT DES HERAUSGEBERS ZUR DEUTSCHEN AUSGABE
}

Die Herausgabe einer deutschen Übersetzung des Lehrbuchs „Mechanik und Molekularphysik" von LandaU, AchiEser und LIFSCHITz bedarf wohl keiner besonderen Rechtfertigung, nach dem das neunbändige Lehrbuch der theoretischen Physik von LANDAU und LIFSCHITZ nun fast vollständig in deutscher Sprache erschienen ist. Das vorliegende Buch stellt eine sehr gelungene Ergänzung des Gesamtkurses der theoretischen Physik dar, indem es eine systematische Ableitung von Beziehungen aus diesem voraussetzt und das Schwergewicht auf die Vermittlurg des Verständnisses für physikalische Zusammenhänge legt. Dies wird anhand eines sehr umfangreichen, zur Mechanik und Molekularphysik gehörenden Stoffes exemplifiziert, so daß dieses Lehrbuch nicht nur für Studenten, sondern auch für Fortgeschrittene und Kenner von erheblichem Wert sein wird.

Es sei noch erwähnt, daß auf Vorschlag der Autoren das letzte Kapitel, das Probleme der Schallausbreitung behandelt, nicht in die deutsche Ubersetzung aufgenommen wurde. Im dritten Kapitel über die Kinematik des starren Körpers sind geringfügige Änderungen vorgenommen worden.

Berlin, im Oktober 1969

F. KASCHLUHN 
